Cahiers $d u$ MONDE RUSSE

\section{Cahiers du monde russe}

Russie - Empire russe - Union soviétique et États indépendants

$43 / 1 \mid 2002$

Varia

\title{
La première édition russe de Palladio par Nikolaj L 'vov et le problème du « vrai goût » palladien.
}

\section{Olga Medvedkova}

\section{OpenEdition}

\section{Journals}

Édition électronique

URL : https://journals.openedition.org/monderusse/8479

DOI : $10.4000 /$ monderusse. 8479

ISSN : $1777-5388$

Éditeur

Éditions de l'EHESS

Édition imprimée

Date de publication : 1 janvier 2002

Pagination : 35-56

ISBN : 2-7132-1773-3

ISSN : $1252-6576$

Référence électronique

Olga Medvedkova, «La première édition russe de Palladio par Nikolaj L'vov et le problème du « vrai goût » palladien. », Cahiers du monde russe [En ligne], 43/1 | 2002, mis en ligne le 01 janvier 2007. consulté le 02 septembre 2022. URL : http://journals.openedition.org/monderusse/8479 ; DOI : https://doi.org/10.4000/monderusse.8479 
chercher : repérer : avancer

Cet article est disponible en ligne à l'adresse :

http://www.cairn.info/article.php?ID REVUE=CMR\&ID NUMPUBLIE=CMR 431\&ID ARTICLE=CMR 4310035

La première édition russe de Palladio par Nikolaj L'vov et le problème du « vrai goût » palladien

par Olga MEDVEDKOVA

\section{Editions de I'EHESS | Cahiers du monde russe}

2002/1 - Vol 43

ISSN 1252-6576 | ISBN 2713217733 | pages 35 à 56

Pour citer cet article :

- MEDVEDKOVA O., La première édition russe de Palladio par Nikolaj L'vov et le problème du « vrai goût " palladien, Cahiers du monde russe 2002/ 1, Vol 43, p. 35-56.

Distribution électronique Cairn pour les Editions de l'EHESS.

(C) Editions de l'EHESS. Tous droits réservés pour tous pays.

La reproduction ou représentation de cet article, notamment par photocopie, n'est autorisée que dans les limites des conditions générales d'utilisation du site ou, le cas échéant, des conditions générales de la licence souscrite par votre établissement. Toute autre reproduction ou représentation, en tout ou partie, sous quelque forme et de quelque manière que ce soit, est interdite sauf accord préalable et écrit de l'éditeur, en dehors des cas prévus par la législation en vigueur en France. Il est précisé que son stockage dans une base de données est également interdit. 


\section{LA PREMIÈRE ÉDITION RUSSE DE PALLADIO PAR NIKOLAJ L'VOV ET LE PROBLÈME DU « VRAI GOÛT » PALLADIEN*}

La fortune de Palladio en Russie ne fut pas moins grande qu'en Angleterre. Elle ne fut pas seulement liée aux deux grands noms de Giacomo Quarenghi et de Charles Cameron, mais était due également à de nombreux architectes russes, parmi lesquels Nikolaj L'vov fut l'un des plus importants. Dans les années 1770-1790, surtout à Moscou, mais aussi à la campagne où se réfugiait la noblesse russe libérée du service obligatoire par le manifeste de 1762, le palladianisme architectural trouva un champ d'application d'autant plus vaste qu'il correspondait profondément à un nouvel idéal de vie. Pourtant ce phénomène n'a jamais fait l'objet en Russie d'une analyse aussi profonde et précise que celle que Rudolf Wittkower ${ }^{1}$ d'abord et plusieurs autres chercheurs ensuite ${ }^{2}$ ont consacrée au palladianisme anglais. Nous ne pouvons citer en effet que quelques articles qui traitent de ce sujet de façon très générale 3 .

\footnotetext{
* Nous avons pu réaliser cette étude grâce à une bourse de l'École française de Rome, en avril 2001.

1. Rudolf Wittkower, Palladio and English Palladianism, Londres, Thames and Hudson, 1974. Dans son livre Wittkower a brillamment démontré l'importance que peut avoir l'étude des publications architecturales pour la compréhension du phénomène d' "acclimatation » d'un style importé. Voir particulièrement les chapitres « English literature on architecture », p. 95112 et « English neoclassicism and the vicissitudes of Palladio's Quattro Libri »,p. 73-94. Voir également : L. Puppi, «Il trattato del Palladio e la sua fortuna in Italia e all'estero », Bollettino CISA, XII, 1970, p. 257-272.
}

2. Voir notamment: Robert Tavernor, Palladio and Palladianism, Londres, Thames and Hudson, 1991.

3. M. Il`in, « Il classicismo russo e il palladianesimo », Bollettino CISA, XII, 1970, p. 183-205; M. Il'in, «O palladianstve D. Kvarengi i N. L'vova » (À propos du palladianisme de Quarenghi et de L'vov), in Russkoe iskusstvo XVIII veka. Materialy $i$ issledovanija ( $L$ 'art russe du XVII siècle. Matériaux et recherches), Moscou, 1973, p. 103-108; André Corboz, «L'architettura neoclassica in Russia », Bollettino CISA, XIII, 1971, p. 274-285 ; I. A. Bartenev, « Il Palladianesimo in Russia », in Palladio : la sua eredita nel mondo. Milan, Electa Editrice, 1980,p. 127-147; 
Dans cette étude, nous nous occuperons d'un seul aspect du palladianisme russe. Aspect ou plutôt événement, car il s'agira de la première - et unique au XVIII siècle - publication russe du premier des Quattro libri sull'architettura de Palladio par Nikolaj L'vov ${ }^{4}$. Comparée à la très riche production éditoriale européenne consacrée au célèbre ouvrage de Palladio, cette unique édition russe peut paraître sans beaucoup d'importance . Le livre ne fut d'ailleurs imprimé qu'en 1798, c'està-dire post factum par rapport à l'activité architecturale liée au modèle palladien qui se déroula en Russie au moins pendant les vingt dernières années du siècle.

Viktor Graščenkov, « Nasledie Palladio v arhitekture russkogo klassicizma » (L'héritage de Palladio dans l'architecture du classicisme russe), Sovetskoe iskusstvoznanie'81, 2, 1982, p. 201-230 ; Natalija Evsina, Russkaja arhitektura v epohu Ekateriny II (L'architecture russe à l'époque de Catherine II), Moscou, 1994, chapitre : « Vkus Palladiev. Palladianstvo v Rossii » (Le goût palladien. Le palladianisme en Russie), p. 92-119. Voir également l'article de Deborah Howard, « Four centuries of literature on Palladio », Journal of the Society of Architectural Historians, 39,3 , octobre 1980 , p. 224-241, dans lequel sont citées les éditions russes de Palladio.

4. Četyre knigi palladievoj arhitektury, v koih po kratkom opisanii pjati Ordenov, govoritsja o tom čto znat’ doľ̌no pri stroenii častnyh domov, dorog, mostov, ploščadej, ristališč i hramov (Les quatre livres de l'architecture de Palladio, dans lesquels après une brève description des cinq ordres, est écrit ce qu'il faut savoir pour la construction des maisons particulières, des routes, des ponts, des places, des xystes et des églises), Saint-Pétersbourg, Šnor, 1798 . Nous avons pu étudier les exemplaires de cette édition qui se trouvent à la Bibliothèque nationale de Russie (Moscou) et à la Bibliotheca Hertziana (Rome). Nous tenons à remercier M. Christoph Frank pour l'aide qu'il nous a accordée à la Bibliotheca Hertziana, ainsi que pour la lecture de cet article.

5. La première tentative pour traduire les Quatre livres de Palladio en russe remonte à l'époque pétrovienne. Il s'agit de la traduction réalisée, en 1699, par le prince Dolgorukov qui séjournait alors à Venise et qui prenait des cours à l'école de Gasparo Vacchia. Cette traduction ne fut pourtant jamais imprimée. Voir: Athitektura cyvil'naja vybrana iz Paladiuša slavnago arhitekta $i j$ iny h mnogih arhitektov slavnyh of matematika $i$ arhitekta Kašpora Vekja pisana $v$ Venecii leta 1699 goda mesjaca sentjabrja učeniem i ť̌čaniem buduči tamo gospodina knjazja Dolgorukova... (L'architecture civile choisie dans le célèbre architecte Palladio et dans d'autres nombreux et célèbres architectes par le mathématicien et architecte Gasparo Vacchia et écrit [traduit] à Venise en l'an 1699 au mois de septembre, par les études et les soins de monsieur le prince Dolgoroukov qui s'y trouvait alors) (Rossijskij Gosudarstvennyj Arhiv Drevnih Aktov à Moscou, cité infra RGADA, f. 181, op. 1, d. 258/463). Pour l'analyse de cette traduction voir : A. A. Tic, « Neizvestnyj russkij traktat po arhitekture» (Un traité d'architecture russe inconnu), in Russkoe iskusstvo XVIII veka. Materialy $i$ issledovanija, Moscou, 1968, p. 17-31. Les gravures de Palladio représentant les escaliers ainsi que certains passages de son traité furent, quelques années plus tard, introduits dans la première édition russe de Vignole, Pravilo o pjati čineh arhitektury... (La règle des cinq ordres de l'architecture...), parue en 1709, puis en 1712. Par ailleurs la bibliothèque de Pierre contenait de nombreuses éditions de Palladio. Le nom de Pierre figure notamment parmi les souscripteurs de l'édition de Palladio par Leoni en 1716. À la fin des années 1730 , la traduction de Palladio fut entreprise par l'architecte Petr Eropkin, ancien pensionnaire de Pierre Ier en Italie, élève de Sebastiano Cipriani. De cette traduction, qui, elle aussi, resta manuscrite, ne s'est conservé que le Quatrième livre (RGADA, f. 16, op. 417, d. 1-28). On peut signaler par ailleurs quelques mentions de Palladio dans les textes russes concernant l'architecture, notamment dans le Mnenie oraznyh pisateljah... (Opinion sur différents auteurs...) (1770), attribué à Baženov, qui place Palladio en tête des architectes modernes, mais qui ne tente pourtant jamais de le faire traduire. (N. Morenec, « Novye materialy o V. I. Baženove » (Nouveaux matériaux sur Baženov), Arhitekturnoe nasledstvo, (Moscou), 1, 1951, p. 102-103). Indirectement liée à Palladio fut en outre la Quatrième ode de Kantemir (années 1730) qui reprenait les thèmes du poème épique de Trissino, l'un des inspirateurs de Palladio. Voir: Valentin Boss, « La quatrième ode de Kantemir et l'Italia liberata de Gian Giorgio Trissino », Cahiers du Monde russe et soviétique, IV, 1-2, 1963, p. 47-55. 
Pourtant il nous semble que cette édition mérite qu'on la considère de plus près. Non seulement parce que, étant donné la pauvreté générale en ouvrages d'esthétique architecturale qui régnait en Russie, chaque édition de ce genre devenait automatiquement un événement, mais encore et surtout parce que le Palladio de L'vov fut réalisé avec un tel soin typographique, avec une telle dépense d'énergie et une si profonde conviction que l'on ne peut lui refuser, même au regard de nombreuses autres éditions européennes de Palladio, d'être l'un des plus beaux Palladio du XVIII siècle. Par ailleurs, cet ouvrage comporte une introduction ainsi qu'un nombre assez important de commentaires qui nous permettent de reconstruire l'attitude intellectuelle et esthétique d'un des représentants les plus cultivés et les plus brillants de la noblesse russe, fasciné par l'art de bâtir.

Au moment où son Palladio voyait le jour, Nikolaj L'vov (1753-1803) avait quarante-cinq ans ${ }^{6}$. Né dans la propriété Čerenčicy (plus tard Nikol'skoe) dans le gouvernement de Tver', au sein d'une famille noble, L'vov perdit tôt son père. Très jeune, il rejoignit Saint-Pétersbourg pour s'inscrire au régiment Izmajlovskij, dont l'école lui offrit une excellente formation, notamment dans le domaine des langues étrangères. Ce fut justement sa connaissance du français, de l'allemand et de l'italien ainsi que quelques relations familiales qui lui ouvrirent les portes du collège des Affaires étrangères, où il entra en 1776 pour y rester jusqu'en 1781 . Mais encore auparavant, en 1773-1775, alors qu'il servait au régiment Preobraženskij, L'vov avait été employé à plusieurs reprises comme courrier, ce qui lui avait permis de visiter l'Allemagne et le Danemark. En 1776-1777, toujours porteur de dépêches, il fit un voyage à Londres, Madrid et Paris, où il s'arrêta pour plusieurs mois (de février à mai 1777). Au cours de ces voyages, L'vov se cultiva, fréquenta les musées, les spectacles, observa les monuments d'architecture. Il se lia par ailleurs d'amitié avec les écrivains Kapnist, Hemnicer, Murav'ev et, à la fin des années 1770, avec Deržavin. Enfin en 1781, L’vov réalisa son voyage d'Italie, dont le journal, entièrement consacré aux arts, fut récemment publié par Konstantin Lappo-Danilevskij . En 1783 L'vov devint membre de l'Académie russe et en 1786 membre honoraire de l'Académie des beaux-arts. Il fut par ailleurs membre de la Société libre d'économie. Au début des années 1790 L'vov abandonna le service et s'installa à Čerenčicy.

Tout au long de sa vie, attiré par un grand nombre d'arts et de sciences musique, dessin, gravure, histoire, géologie - ainsi que par différentes techniques,

6. Sur L'vov voir: M. V. Budylina, O. I. Brajceva, A. M. Harlamova, Arbitektor N. A. L'vov, Moscou, 1961 ; N. I. Nikulina, Nikolaj L'vov, Leningrad, 1971 ; A. N. Glumov, N. A. L'vov, Moscou, 1980 ; N. A. L'vov, Izbrannye sočinenija (Eurres choisies), préface de D.S. Lihačev, éd. K.Ju. Lappo-Danilevskij, Cologne, Böhlau, 1994. Dans tous ces ouvrages, l'année 1751 est indiquée comme la date de naissance de L'vov. La célébration à Saint-Pétersbourg et à Tver' du $150^{e}$ anniversaire de L'vov en 2001 a donné lieu à une exposition et à plusieurs colloques. Les travaux suscités par le jubilé ont permis de déterminer sa véritable date de naissance, soit deux ans plus tard qu'on ne l'avait cru. La nouvelle célébration est donc programmée maintenant pour l'an 2003.

7. N. A. L'vov, Ital'janskij dnevnik (Journal italien), éd. K. Ju. Lappo-Danilevskij, Cologne, Böhlau, 1998. 
L'vov privilégia particulièrement la littérature et l'architecture. Le rôle des voyages et surtout de son voyage italien dans sa formation d'architecte, par ailleurs dilettante, fut sans doute décisif. Comme l'écrivait plus tard son ami Murav'ev :

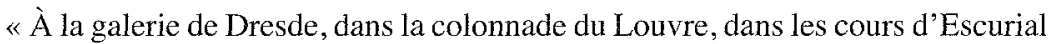
et, enfin, à Rome, patrie des arts et des antiquités, il puisait ces formes majestueuses, cette notion de simplicité (ponjatic prostoty), cette harmonie (sorazmernost) inimitable, qui respirent dans les ouvres magnifiques des Palladio et des Michel-Ange. $»^{8}$

L'vov commença à pratiquer l'architecture juste avant son départ pour Rome, en 1780. Son premier projet réalisé fut celui de la cathédrale Saint-Joseph qui devait commémorer la rencontre entre Catherine II et Joseph II, rencontre qui déboucha sur une alliance entre la Russie et l'Autriche dans la guerre contre la Turquie. La construction du monument fut précédée d'un concours que L'vov remporta grâce à la protection du chancelier Bezborodko, l'un des auteurs du «projet grec » dans la politique russe ${ }^{9}$. La cathédrale fut conçue comme une réplique de la Sainte-Sophie de Constantinople. Pourtant ce prototype fut «traduit » dans les formes de l'architecture grecque antique. Cette combinaison devait justifier la prétention des Russes, à la fois derniers Grecs et derniers Byzantins, au trône de la Grèce moderne ${ }^{10}$. Pour faire «à la grecque », L'vov utilisa dans son projet des portiques doriques et des colonnes sans bases car, comme il l'écrivait lui-même, les bases «n'ont jamais été utilisées dans cet ordre dans la meilleure époque de l'architecture grecque, comme en témoignent toutes les ruines d'Athènes, ainsi que les temples de Paestum. » ${ }^{i l}$ En même temps, la coupole de la cathédrale s'inspirait de celle du Panthéon, mais aussi, par sa double voûte, de la coupole de l'église Saint-Pierre à Rome.

Catherine II accepta sans doute le projet de L'vov grâce à sa référence grécobyzantine qui s'inscrivait dans le contexte idéologique du moment. Quant à L'vov, il put démontrer par ce projet sa capacité à réaliser une parfaite adaptation du

8. Rossijskaja Publičnaja Biblioteka, Saint-Pétersbourg, manuscrits, fonds Olenin, $\mathrm{n}^{\circ} 760$; publié dans : N. A. L'vov, Izbrannye sočinenija, op. cit., p. 361.

9. Voir sur le « projet grec » et son influence sur les arts et les lettres en Russie : Andrej Zorin, Kormja dvuglavogo orla... Literatura i gosudarstvennaja ideologija $v$ Rossii v poslednej treti XVIII - pervoj treti XIX veka (En nourrissant l'aigle bicéphale... La littérature et l'idéologie de I'État en Russie au dernier tiers du XVIII' et au premier tiers du XXXe siècles), Moscou, NLO, 2001 , chapitre I.

10. Sur l'idée de construire la « nouvelle Constantinople », notamment à Carskoe Selo, voir : Dmitrij Švidkovskij, « Poetika arhitektury v russkom dvorcovo-parkovom ansamble epohi Prosveščenija » (La poétique de l'architecture dans les ensembles russes des palais et jardins de l'époque des Lumières), in Hudožestvennye modeli mitozdanija (Les modèles artistiques du monde), Moscou, NII RAH, 1997, p. 209-222 ; Id., « Prosveščenie i russkij sad vtoroj poloviny XVIII veka » (Les Lumières et le jardin russe dans la seconde moitié du XVIII ${ }^{e}$ siècle), in Istorija sadov, (Moscou), 1, 1994, p. 36-43.

11. Le projet fut gravé en 1782 avec les explications de L'vov. Cité d'après N. I. Glinka, « Nikolaj L'vov », in Zodčie Sankt-Peterburga. XVIII vek (Les architectes de Saint-Pétersbourg au XVIII siècle), Saint-Pétersbourg: Lenizdat, 1997, p. 835. 
langage architectural qui tirait toute sa valeur et sa force «parlante » du mélange savant entre un certain nombre d'éléments empruntés aussi bien à l'antiquité qu'à l'architecture moderne. L'heureuse idée d'utiliser le portique manifestement grec par l'emploi des colonnes doriques sans bases n'aurait sans doute pu lui venir à l'esprit sans la connaissance de certaines publications qui parurent à la fin des années 1750 et au début des années 1760 , consacrées aux antiquités de la Grèce et de Paestum ${ }^{12}$. Ainsi, autre enseignement de cette histoire, pour la première fois en Russie, un dilettante éclairé donnait une leçon d'architecture aux professionnels grâce à ses connaissances livresques.

Parmi les livres d'architecture qui se trouvaient dans la bibliothèque de L'vov, deux se sont conservés : une édition des cahiers de Neufforge ${ }^{13}$ et une Théorie de l'art des jardins de Hirschfeld ${ }^{14}$. Dans les deux ouvrages on trouve une quantité de notes marginales de L'vov ainsi que ses dessins collés entre les pages, qui témoignent d'une lecture aussi attentive que créative. De même, avant de partir pour l'Italie, L'vov entreprit la lecture de Winckelmann. Le 9 mars 1781, peu avant son départ, il note, dans son cahier de travail, des citations de Winckelmann, tirées de l'édition française ${ }^{15}$. Dans son Journal italien l'influence des idées de Winckelmann est parfaitement reconnaissable, renforcée probablement par certaines rencontres qu'il fit pendant ce voyage et notamment celle de Johann Friedrich Reiffenstein, un des amis les plus proches de Winckelmann et l'agent artistique de Catherine II à Rome ${ }^{16}$. Cette influence est particulièrement évidente dans la description du groupe de Niobé, que L'vov a vu à Florence ${ }^{17}$. À l'émerveillement

12. Il s'agit des deux publications sur les antiquités de la Grèce de Le Roy et de Stuart et Revett : Julien-David Le Roy, Les ruines des plus beaux monuments de la Grèce, Paris, H. L. Guerin \& L.F. Delatour, Jean-Luc Nyon, Amsterdam, Jean Neaulme, 1758 ; James Stuart, Nicholas Revett, The Antiquities of Athens. Measured and delineated by James Stuatt and Nicholas Revett, painters and architects, Londres, John Haberkorn, vol. 1, 1762, ainsi que de plusieurs (non moins de huit) publications sur Paestum, parues entre 1764 et 1784. Sur les publications des antiquités de Paestum voir $\mathrm{S}$. Lang, « The early publications of the temples at Paestum », Journal of the Warburg and Courtauld Institutes, 13, 1950, p. 48-64. L'histoire du «Doric revival » est étudiée dans : N. Pevsner, S. Lang, « Apollo or Baboon », Architectural Review, dec. 1948. Voir également le catalogue de l'exposition : La fortuna di Paestum e la memoria moderna del dorico, 1750-1830, Florence, 1986, vol. 1-2.

13. J.-F. Neufforge, Recueil élémentaire d'architecture, t. 1-8, Paris, 1757-1777. L'exemplaire de L'vov se trouve au Musée du château de Gatčina.

14. Christian Cay Lorenz Hirschfeld, Theorie des Gartenkunst, Leipzig, Weidmanus Erben und Reich, 1775 (édition en 5 vol., même éditeur, 1779-1785; trad. fr. : Théorie de l'art des jardins, même éditeur, 5 vol., 1779-1785). L'exemplaire de L'vov se trouvait jusqu'en 1967 à la bibliothèque du Musée des beaux-arts Pouchkine à Moscon.

15. Histoire de l'art chez les anciens. Par Mr. J. Winckelmann... Ouvrage traduit de l'allemand par G. Sellius et rédigé par G. B. R. Robinet, Paris, 1766. La citation est relevée par Ju. K. Lappo-Danilevskij, «Ob "Ital' janskom dnevnike" N. A. L'vova » (À propos du Journal italien de L'vov), in N. A. L'vov, Ital'janskij dnevnik, op. cit., p. 36-37.

16. Sur Reiffenstein voir notamment: Christoph Frank, "Plus il y en aura, mieux ce sera". Caterina II di Russia e Anton Raphael Mengs. Sul ruolo degli agenti "cesare," Grimm e Reiffenstein », in Mengs : la scoperta del neoclassico, a cura di Steffi Roettgen, catalogue de l'exposition (Padoue, 2001), Venise, Marsilio, 2001, p. 87-95.

17. N. A. L'vov, Ital'janskij dnevnik, op. cit., p. 68. 
devant cette œuvre dans laquelle, à la suite de Winckelmann, il voit « l'exemple de la perfection de la sculpture grecque [...] d'un style suprême » se mêle un credo de sa nouvelle foi, très proche effectivement de celui de Winckelmann et de Reiffenstein $^{18}$. La remarque sur la beauté des draperies antiques est particulièrement significative: «[...] la draperie (draperi) est merveilleuse; Mr Falconet ne l'approuve pas dans son ouvrage, ce qui n'est pas étonnant, car sa simplicité est parfaitement à l'opposé des sculpteurs français de l'école de Bernini, dont tous les drapés sont gonflés par le vent $[. ..]{ }^{19}$. Depuis Perrault jusqu'à Falconet, la supériorité des draperies chez les sculpteurs modernes fut l'un des arguments de la théorie française contre l'autorité de l'Antiquité. Le passage cité du journal de L'vov reprend à la lettre la critique des positions esthétiques de Falconet que l'on trouve dans l'Histoire de l'art chez les Anciens de Winckelmann ${ }^{20}$. Dans plusieurs autres passages, L'vov affirme la suprématie de l'art ancien sur le moderne et ne fait exception que pour quelques artistes: Raphaël, Michel-Ange et, parmi les contemporains, Mengs, ce « nouveau Raphaël ».

Quant à l'architecture, elle n'apparaît que très peu dans le Journal. Pourtant le nom de Palladio y figure déjà. À Venise, L'vov remarque l'église de San Giorgio Maggiore. Plus tard, dans son édition de Palladio il racontera que ce fut justement à Venise qu'il avait acheté l'ancienne édition des Quattro libri. En effet dès son retour d'Italie, la référence palladienne devient de plus en plus présente dans ses projets, tels que le projet de l'Hôtel des postes à Pétersbourg qui date de 1782 ou, trois ans plus tard, le projet de l'église Saints-Boris-et-Gleb de Toržok, en même temps que plusieurs résidences et hôtels particuliers à Moscou, à Pétersbourg et en province.

Ainsi, aux début des années 1780 , par ses voyages, ses rencontres, ses lectures, L'vov se forme dans le domaine des arts et se transforme progressivement en une sorte d'arbitre du goût pour son entourage, dans lequel on trouve les meilleurs écrivains et artistes de l'époque. En effet, dans les souvenirs de ses amis, il apparaît comme un «homme de goût» par excellence. Son cousin Fedor L'vov le décrit comme « le génie du goût (vkus), qui apposait son sceau sur les œuvres de ses amis. ${ }^{21}$ De même Deržavin écrit :

«Il était plein d'intelligence et de connaissances, aimait les sciences et les arts et se distinguait par un goût raffiné et supérieur ( vozvyšennyj), grâce auquel aucun défaut, aucune qualité d'une œuvre artistique ou littéraire ne pouvaient lui échapper. Les hommes qui pratiquaient les lettres, les différents arts et même les

18. Voir, par exemple : Pascal Griener, L'esthétique de la traduction: Winckelmann, les langues et l'histoire de I'art (1755-1784), Genève, Droz, 1998 (Histoire des idées et critique littéraire, 373 ).

19. N. A. L'vov, ltaljans kij dnevnik, op. cit., p. 68.

20. Histoire de l'art chez les anciens. Par Mr. J. Winckelmann..., op. cit, partie 1, chapitre 4. Winckelmann renvoie aux Réflexions sur la sculpture de Falconet (Paris, Prault, 1761).

21. F. P. L'vov, « Biografii rossijskih pisatelej. N. A. L'vov» (Biographies d'écrivains russes. N. A. L'vov), Syn otečestva, 77, 1822,p. 177. 
métiers recouraient souvent à ses conseils, et son jugement faisait souvent loi. $\gg 22$

Comment peut-on comprendre cette notion de goût, que L'vov lui-même employait si souvent ? Était-ce une construction intellectuelle consciente, un « programme » ou seulement un vague ensemble d'appréciations et d'avis ? De prime abord cette notion semble venir du vocabulaire français. En effet l'allégorie du goût apparaît dans presque tous les portraits des grands amateurs d'art français de l'époque, notamment dans celui de Mariette gravé par Cochin ou dans celui de Cochin, gravé par Gravelot ${ }^{23}$. Le génie du Goût devient, à la fin du XVIII ${ }^{e}$ siècle, comme une marque particulière française, en partie grâce à Voltaire. Dans l'article « Goût » du volume 7 de l'Encyclopédie, Voltaire définit le goût dans les arts par rapport au goût physiologique. Le goût artistique est un «discernement prompt » qui reconnaît rapidement, par la voie la plus courte, ce qui est beau, de même que la langue reconnaît ce qui est bon. Venu directement de la nature, le vrai goût s'attache à ce qui est simple et naturel. La préférence pour ce qui est trop recherché, étudié, pour le burlesque, le précieux et l'affecté est une sorte de maladie de l'esprit. Il y a donc un bon goût et un mauvais goût. Le bon peut se former chez un particulier, bien sûr, mais surtout au sein d'une nation. Sans mentionner la France, Voltaire décrit bien évidemment le bon goût d'une nation par référence à l'expérience française ${ }^{24}$.

Si une nation atteint le bon goût, elle est en droit de juger les autres. Si le goût est dans la nature, ce jugement a une valeur universelle. Ainsi 1'Europe peut-elle juger l'Asie et la France les autres pays de l'Europe ${ }^{25}$. Ce droit de juger que possède le détenteur du goût le plus naturel et donc le plus vrai appelle une sorte de dictature du goût. L'homme de goût ne reconnaît aucune autorité, sa position est celle du critique libre qui regarde les ouvrages des Anciens d'un même ceil que ceux des

22. Gavrila Deržavin, $O b$ ”jasnenija na sočinenija Deržavina, im samim diktovannye (Explications des cuvres de Deržavin, dictées par lui-même), Saint-Pétersbourg, 1834, p. 60-61. Je remercie M. Jean Breuillard d'avoir lu cet article et attiré mon attention sur la nouveauté relative de la notion de «goût » qui n'apparaît en Russie, dans sa signification esthétique, qu'au milieu du XVIII siècle.

23. Frontispice du livre Iconologie par Figures ou Traité complet des Allégories, Emblèmes \&c. Ouvrage utile aux Artistes, aux Amateurs, et pouvant servir à l'éducation des jeunes personnes. Par MM. Gravelot et Cochin, Paris, Le Pan, 1791, t. 1. Dans l'explication de ce frontispice nous lisons : «Les grâces ornent de guirlandes le buste de Cochin ; la muse de l'histoire consacre le nom de cet artiste dans ses fastes, \& tandis que le génie du dessin indique les productions de Cochin, le dieu du goût dépose sur son buste la couronne réservée à ceux qu'il inspire. $\gg(\mathrm{p} . \mathrm{I})$.

24. Voltaire, «Goût », in Encyclopédie ou Dictionnaire raisonné des sciences, des arts et des métiers, vol. 7, Paris, 1757 [reprint 1966], p. 761.

25. Ibid., p. 761. Si Montesquieu, dans son «fragment sur le goût»» joint à l'article de Voltaire, se livre à un développement philosophique beaucoup plus subtil, il n'en est pas moins strict dans son exposé des règles du bon goût. Ainsi dans le domaine de l'architecture, il ne mentionne que celle des Grecs « qui a peu de divisions \& de grandes divisions » et qui, par conséquent, « imite les grandes choses... ( (Ibid, p. 764). Nous ne serons pas surpris de trouver la citation de cet article dans l'ouvrage de David Le Roy consacré aux antiquités grecques : J .D. Le Roy, op. cit.. 
Modernes. Dans ses Observations sur les antiquités de la ville d'Herculanum, publiées en $1756^{26}$, Charles-Nicolas Cochin est peut-être le plus critique de tous les auteurs français. Il exalte sa liberté de jugement face aux Anciens en émaillant ses descriptions de remarques dignes du Diderot des Salons.

Or la notion de goût chez L'vov, telle qu'il la formule notamment dans sa préface de l'édition bilingue d'Anacréon ${ }^{27}$, ne ressemble nullement à celle de Voltaire. Cette préface est pour nous d'autant plus intéressante qu'elle est très proche de celle dont L'vov dotera quatre ans plus tard son édition de Palladio. En effet les deux langages, littéraire et architectural, semblent composer pour L'vov les deux faces d'un seul et unique langage humain. Ainsi, dans un des commentaires, $L^{\prime}$ vov observe qu'Anacréon écrivait en langue « dorique ${ }^{28}$.

Selon ses propres aveux, L'vov s'est formé un goût artistique à la suite de sa découverte de l'art et de la littérature antiques. Cette découverte lui « a purifié le goût », l'a détourné des faux attraits des poètes français modernes et lui a fait sentir « la grave et simple beauté de la vérité » (važnuju i prostuju krasotu istiny) ${ }^{29}$. Ainsi à la différence de Voltaire, chez qui le goût naît d'un sentiment naturel, le goût de L'vov est le résultat de sa découverte de l'antiquité.

C'est de cette découverte que découle ensuite le sentiment de l'authenticité (et non l'inverse !), qui pénètre tous les écrits de $L^{\prime}$ vov. Selon lui, seul celui qui puise à la source au lieu d'imiter les imitateurs est capable de créer des cuvres originales. On sent ici l'héritage néoplatonicien; l'antiquité se confond avec la beauté idéale.

Enfin ce goût ancien, vrai et authentique, est aussi naturel par excellence, car 1 'art antique est celui qui nous transmet « le vif et tendre sentiment de la nature ${ }^{30}$.

Le goût représente pour L'vov une notion d'autant plus sérieuse qu'elle est connotée par tout un ensemble de convictions sociales, morales et nationales. En effet le goût est pour lui une qualité aristocratique (L'vov met particulièrement en valeur l'origine non seulement noble, mais royale d'Anacréon). L'homme de goût est vertueux, il échappe plus facilement aux péchés, car il est protégé par le sentiment de la honte mais surtout par le dégoût (otvraščnie) ${ }^{31}$. Enfin ce goût naturel est parfaitement adapté au caractère de la nation russe, qui est très proche, d'une part, de la nature et, d'autre part, de la Grèce. Ainsi la traduction du grec en russe produit l'effet de l'original et se distingue des fioritures à la française.

26. Charles-Nicolas Cochin, Observation sur les antiquités de la ville d'Herculanum avec quelques réflexions sur la peinture et la sculpture des Anciens; \& une courte description de quelques antiquités des environs de Naples, Paris, 1756. Voir également: Christian Miche], Charles-Nicolas Cochin et l'art des Lumières, Rome, EFR, 1993.

27. [N. A. L'vov], Stihotvorenija Anakreona Tijskogo. Perevel *** (Poèmes d'Anacréon. Traduit par***), Saint-Pétersbourg, 1794.

28. Cité d'après l'édition: N. A. L'vov, Izbrannye sočinenija, op. cit., p. 143 (commentaires sur le $2^{\mathrm{e}}$ livre).

29. Ibid., p. 108.

30. Ibid., p. 107.

31. Ibid., p. 110. 
À partir de ces idées il nous sera maintenant plus facile de comprendre le «Palladio russe » de L'vov. Dans sa préface il représente justement Palladio comme l'incarnation d'un « goût pur », qu'il faut puiser à sa source italienne. Pour la première fois en Russie, L'vov publie une édition architecturale bilingue russoitalienne. Cette circonstance même fait apparaitre le livre comme un véritable manifeste de la nouvelle orientation « italienne » contre l'orientation française. La préface de L'vov s'ouvre d'ailleurs par la citation du nom d'Algarotti qui, dans son Essai sur l'Académie de France qui est à Rome ${ }^{32}$, appelle Palladio le « Raphaël de l'architecture ». Or ce texte d'Algarotti est particulièrement critique à l'égard de la théorie esthétique française.

Le frontispice ${ }^{33}$ du livre [ill. 1] représente un piédestal sur lequel figurent le titre original des Quattro libri, l'année de leur parution et, plus bas, le titre russe, ainsi que le nom de l'éditeur L'vov. Alors que la plupart de ses éditions étaient anonymes, L'vov affiche fièrement son nom dans cet ouvrage d'architecture, publie son portrait ${ }^{34}$ à la suite du frontispice [ill. 2] et signe l'introduction. Cette attitude est proche de celle de lord Burlington qui, lui aussi, en 1730, a publié les dessins de Palladio avec son propre portrait sur la page de titre ${ }^{35}$ [ill. 3]. De façon générale, le type culturel d'un «nobleman-architect » représenté par Burlington, aurait pu servir de modèle à $L^{\prime}$ vov, bien qu'il ne cite jamais son nom ${ }^{36}$.

Toujours dans le frontispice, un amour coiffé d'une couronne d'étoiles érige sur le piédestal un médaillon représentant le buste de Palladio et le place à côté d'un autre médaillon représentant saint Georges, le protecteur de la Russie : tous deux se retrouvent sous les ailes protecteurs de l'aigle. Le dieu du temps, Saturne, qui tient

32. Francesco Algarotti, Essais sur la peinture et sur l'Académie de France établie à Rome, traduit de l'italien par M. Pingeron, Paris, Merlin Libraire, 1769 [Genève, Minkoff reprints, 1972].

33. On peut lire sur la gravure: «Inventé par Ivanoff, dessiné par A. Olenin et gravé par Mayr ».

34. Le portrait de L'vov est peint par Dmitrij Levickij, ami de L'vov, et gravé par Aleksandr Gardeev. L'original du portrait (1770 ?) se trouve au Musée de la littérature à Moscou, inv. X-836.

35. Burlington, Fabbriche Antiche disegnate da Andrea Palladio Vicentino e'date in luce da Riccardo Conte di Burlington, Londres, 1730. Par ailleurs dans le frontispice de son ouvrage sur les bains des Romains, paru en 1772, Carles Cameron reprend tout le cadre du frontispice de Burlington en remplaçant le portrait de ce dernier par le buste de Palladio: Charles Cameron, The baths of the Romans explained and illustrated: with the restorations of Palladio corrected and improved. To which is prefixed an introductory preface, pointed out the nature of the work; and a dissertation upon the state of the arts during the different periods of the Roman empire. Description des bains des Romains, enrichie des plans de Palladio, corrigés et perfectionnés ; et précédée d'une préface, en forme d'introduction, sur la nature de cet ouvrage, et d'une dissertation sur l'état des arts, durant les différentes périodes de l'Empire Romain; en anglais et en français, Londres, S. Leacroft, 1772 (réédité en 1774 et 1775) [ill. 4]. Nous pouvons ainsi voir l'importance des messages que les éditeurs des traités « cachent » dans leurs frontispices, de même que la relation étroite qui existe entre ces gravures.

36. Par ailleurs L'vov connaît l'ouvre de Kent dont il cite le nom dans son explication pour le projet du jardin du comte Bezborodko à Moscou, publié dans : G. G. Grimm, « Proekt sada Bezborodko v Moskve (materialy k izučeniju tvorčestva N. A. L'vova) » (Le projet du jardin de Bezborodko à Moscou (Matériaux pour une étude de l'œuvre de L'vov), Soobščnija instituta istorii iskusstv, 4-5, 1954, p. 107-135. 
dans sa main un sablier, s'allonge sur le piédestal à côté du portrait de Palladio. Au pied du piédestal, le génie du Goût invite à contempler ce portrait. Une autre figure allégorique représente l'Architecture, vêtue d'un costume qui tient à la fois d'un costume grec ancien et d'un costume russe national. Elle est assise sur le chapiteau d'une colonne antique, un niveau de maçon à la main. À côté, deux putti s'amusent à tracer un dessin d'architecture ; le plus petit dessine, alors que le grand lui montre du doigt l'effigie de Palladio.

Cette composition allégorique n'est pas difficile à déchiffrer ${ }^{37}$. Elle représente 1'instauration en Russie d'un véritable culte de Palladio ${ }^{38}$. Le Temps (qui d'ailleurs apparaît également, mais en marge, dans le frontispice de l'édition originale de Palladio [ill. 6]) découvre la gloire de l'architecte, rappelant une représentation allégorique du Temps Révélateur, ou encore du Temps et de la Vérité, qui restait dans ses différentes variantes assez courante au XVIII ${ }^{\mathrm{e}}$ siècle $^{39}$. Dans la composition de L'vov, le portrait de Palladio fait figure de la Vérité, longtemps cachée et enfin découverte. De même, dans le frontispice dessiné par Sebastiano Ricci pour l'édition anglaise de Palladio de 1716, réalisée par Giacomo Leoni ${ }^{40}$, un Saturne tire le rideau pour montrer le buste de Palladio. On retrouve par ailleurs ce motif le rideau tiré derrière lequel le spectateur découvre la vérité - dans le frontispice du traité de perspective italien traduit et édité par L'vov en $1788^{41}$. La « vérité » qui apparaît derrière le rideau est représentée par un bâtiment très ressemblant à la villa Rotonda de Palladio. Dans l'édition de Leoni, Brittania assise à côté du portrait de Palladio montre les armes de l'Angleterre. Dans l'édition de L'vov, l'allégorie de

37. Du moins dans sa signification la plus évidente. Étant, comme beaucoup de ses amis, membre actif de l'ordre des francs-maçons, L'vov était très versé dans l'art des allégories. L'un des programmes allégoriques auxquels il participa fut celui du portrait de Catherine II en législatrice réalisé pour Bezborodko par Levickij (1783, Musée Russe, Saint-Pétersbourg) dans lequel apparaissaient notamment les signes astrologiques. Dans son portrait publié dans Palladio nous pouvons voir notamment, en bas, les branches d'acacia croisées avec un compas, ce qui pouvait être une allusion à l'ordre. Il est fort probable que l'amour à couronne d'étoiles n'était pas sans rapport avec l'Astrologie. Par ailleurs la couronne d'étoiles signifiait l'immortalité dans certaines gravures maçonniques. Ainsi on pourrait également interpréter le frontispice de la façon suivante : l'effigie de Palladio est révélée par le génie de l'immortalité et/ou associée aux sphères célestes.

38. L'idée de mettre le portrait de l'architecte sous les armes de la Russie n'était pas nouvelle. Déjà, dans le frontispice de la première édition de Vignole en russe, l'aigle bicéphale se trouve en haut de la composition. Vignola, Pravilo o pjati čineh arhitektury Jakova Barocija Devignola. Poveleniem Velikago Gosudarja Carja i velikago knjazja Petra Alekseeviča Samoderžca Vserossijskago; Grydyrovana i napečatana... (La règle des cinq ordres de l'architecture de Vignole. Par la volonté du Grand Souverain. Tsar et Grand Prince Petr Alekseevič, Autocrate de toute la Russie ; Gravé et imprimé...), Moscou, 1709 [il1.5].

39. Voir : Erwin Panofsky, « Le Vieillard Temps », in Essais d'iconologie. Thèmes humanistes dans I'art de la Renaissance, trad. fr., Paris, Gallimard, 1967,p. 105-150 ; Friz Saxl, « Veritas Filia Temporis », in Philosophy and History, Essays presented to Ernest Cassirer, Oxford, 1936, p. 197-222.

40. Sur cette édition voir : Rudolf Wittkower, « English neoclassicism and the vicissitudes of Palladio's Quattro Libri. », op. cit., p. 73-94.

41. Il s'agit d'une édition trilingue d'un livre italien de perspective. Razsuždenie o prospektive $\checkmark$ pol'zu narodnyh učilišč, izdal $N$. L vov, sent. 101788 goda. (Considérations sur la perspective pour le profit des écoles publiques, publié par N. L'vov le lO septembre 1788). 
l'Architecture se confond, à cause de son costume, avec celle de la Russie qui se rapproche à son tour de celle de la Grèce antique. Autre remarque, dans l'édition de Leoni, Palladio est représenté en jeune homme : ce portrait fut inventé par l'auteur pour donner d'avantage de charme à son édition. Ce même portrait du jeune Palladio apparaît dans l'un des dessins préparatoires de L'vov pour le frontispice de son édition. Or, dans la version définitive, il le remplace par le «vrai » portrait stylisé à l'antique (les yeux sans pupilles!), qu'il copie dans l'édition de Bertotti Scamozzi [ill. 7].

Le frontispice de L'vov ne nous surprend en fait que par un seul détail, à savoir par la date de l'édition palladienne indiquée sur le piédestal. Dans sa préface, après avoir énuméré plusieurs éditions palladiennes françaises, anglaises et italiennes, notamment celles de Roland Fréart, de Le Muet, de Leoni, et de Scamozzi, L'vov écrit :

« [...] mais en même temps, un petit nombre d'amateurs de l'Architecture pure, les connaisseurs et les artistes, attirés davantage par la beauté et la précision des profils que par le prix élevé de ces éditions, recherchaient la vieille édition (Edicija) de Palladio, publiée à Venise en 1616 par Carampello, gravée sur bois mais corrigée par l'Auteur même. Cette édition devint enfin si rare et si chère, qu'un certain libraire avisé trouva son compte à la réimprimer et à reproduire l'original en imitant exactement ses défauts, qui apparurent sur les planches de bois à cause de leur usage ; mais il ne pouvait imiter les vraies beautés (dejstvitel'nye krasoty), que l'artiste aperçoit dans l'original. Cependant, même ces enfants de la cupidité, sous le faux nom de leur père célèbre, se vendaient et continuent à se vendre assez cher. Les bibliomanes achètent parfois une fausse édition au papier bruni et aux bordures rongées, dans laquelle le connaisseur ne retrouve point l'Auteur. Pendant mon séjour à Venise, j'eus l'occasion, lors d'une vente publique d'une grande et ancienne bibliothèque, d'acheter assez cher l'édition originale de Carampello [...]. » ${ }^{42}$

Apparemment bien renseigné sur les différentes éditions de Palladio, à tel point qu'il cite même la « fausse édition », c'est-à-dire le reprint de l'édition de 1570 publié par Pasquali en 1768, L'vov prend celle de 1616 pour l'édition originale de Palladio. Or la date de la première parution des Quattro libri - 1570 - ainsi que le nom d'éditeur - Franceschi - étaient bien sûr parfaitement connus de tous les palladianistes. L'édition de 1616 n'était en réalité que la quatrième, faite sur les planches de bois originales, bien que déjà assez usées. Ainsi le «voyage initiatique » de L'vov à Venise et l'achat du livre « original » qui rappelle celui de Burlington, qui, lui, possédait dans sa bibliothèque dix exemplaires du livre dont cinq de la première édition, était quelque peu compromis par son erreur sur la date $^{43}$.

42. «Ot izdatelja ruskago Palladija » (De la part de l'éditeur du Palladio russe), in Četyre knigi palladievoj arhitektury, op. cit., non paginé.

43. Il existe une autre édition de Palladio du XVIII ${ }^{e}$ siècle, celle de William Halfpenny, dans laquelle l'auteur indique, probablement en se trompant, l'année 1581 comme la date de la première parution, édition qui était « snobée » par le milieu érudit. Andrea Palladio's first Book of Architecture, Corrected from his Original Edition printed at Venice, 1581 (1751), voir R. Wittkower, op. cit., p. 90. 
Cette erreur, dont nous n'avons trouvé aucune explication, est d'autant plus surprenante que dans sa préface L'vov insiste justement sur l'authenticité de sa publication qui remonte à la source. Il écrit :

«[...] j’ai employé près de huit ans pour la remettre en ordre ; complétant les défauts des planches de bois en les comparant avec les autres éditions sur cuivre, j'ai dessiné les quatre livres de l'Architecture de Palladio, ce qui représente plus de 200 dessins, complètement semblables à l'original y compris dans ses mesures, sans rien changer ni ajouter, et j'édite Palladio avec toute l'authenticité que mérite sa perfection. $\gg^{44}$

Ce désir d'authenticité rappelle l'objectif que poursuivait le traducteur français de Palladio, Fréart de Chambray, qui opposait sa « vraie » édition à celle de Le Muet ${ }^{45}$. Il est également proche de l'attitude des architectes-éditeurs anglais de Palladio, du cercle de Burlington, tels que Colin Campbell (1728) et Isaak Ware (1738)46, qui s'opposaient à l'édition de Leoni. Mais, dans le contexte russe, il s'agissait de la première édition nationale de Palladio. La seule référence russe implicite que nous pouvons relever dans ce manifeste de l'authenticité renvoie à l'édition de Vitruve par Baženov et Karžavin qui parut peu avant l'édition de L'vov et qui représentait une traduction à partir de l'édition française de Claude Perrault ${ }^{47}$. Mais pour L'vov, il était visiblement question de dépasser en authenticité toutes les éditions

44. «Ot izdatelja ruskago Palladija », art. cit. L'vov réalisa un ensemble de 109 dessins pour les livres III et IV de Palladio (Naučno-Issledovatel'skij Muzej Akademii Hudožestv NIMAH, Saint-Pétersbourg). Avec les dessins gravés pour le livre I, il y avait en tout 201 dessins. L'hypothèse de Uvarova selon laquelle les dessins furent réalisés non par L'vov mais par Ivan Tupylev ne nous paraît pas convaincante. (N. I. Uvarova, « $O$ tom, čego ne

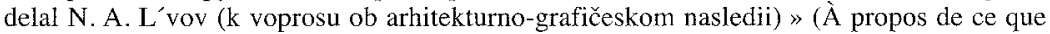
n'a pas fait N. A. L'vov (la question de l'héritage architectural et graphique)), in Giacomo Quarenghi i neoklassicizm XVIII veka, actes du colloque, Ermitage, Saint-Pétersbourg, 1994, p. 54-57).

45. Dans son édition, Fréart de Chambray utilise deux fois le frontispice de l'édition originale de Palladio, avec le texte italien d'abord, français ensuite, ce qui signifiait sans doute qu'en changeant de pays et de langue Palladio restait fidèle à lui-même.

46. Sur la page de titre de The four books of architecture by Andrea Palladio (1738) de Ware on lit : « Literally translated from the original Italian and further Particular Care has been taken to preserve the Proportions and Measures from the Original, all the Plates being Engraved by the Autor's own hand. » (Voir R. Wittkower, op. cit, p. 88). À comparer avec l'attitude de Leoni qui écrivait : " [...] that the Work may in some sort be rather considered as an Original, that an Improvement. » (Ibid., p. 85). Il ne faut pas oublier que Charles Cameron qui arrive en Russie en 1779 fut l'un des souscripteurs du livre de Ware. Voir sur Cameron : Dimitri Švidkovskij, The Empress and the Architect, New Haven - Londres, Yale University Press, 1996.

47. Sokraščennyj Vitruvij ili soveršennyj Arhitektor. Perevod Arhitektura-pomoščnika Fedora Karžavina (Abrégé de Vitruve ou l'architecte parfait. Traduit par l'architecte adjoint Fedor Karžavin), Moscou, imprimerie de l'Université de Novikov, 1789 ; Marka Vitruvija Polliona ob Arhitekture, kniga pervaja i vtoraja s primečanijami doktora medicyny i Francuzskoj Akademii člena g. Pero [...] (De l'Architecture de Marc Vitruve Pollio, livre un et deux avec les remarques du docteur en médecine et membre de l'Académic française $M$. Perrault), SaintPétersbourg, Académie Impériale des Sciences, 1790. 
européennes. En effet, il oppose le palladianisme russe aux variantes française et anglaise désignées comme non authentiques.

«Je voulais que, dans mon édition, Palladio ressemblât à lui-même et non à un Français qui eût chargé son goût pur par des décorations frisottantes, ou encore à un Anglais qui eût fait reposer ses graves beautés sur des allumettes [il évoque, bien sûr, les colonnes, O. M.], tous deux voulant contenter le goût de leur patrie. Que le goût Palladien soit dans ma patrie, car les boucles françaises et la finesse anglaise ont déjà assez d'imitateurs. »48

Ainsi en retournant vers la source, les Russes devaient atteindre l'original dans sa qualité universelle et générale, sans se laisser séduire par ses versions nationales. Or, comme on s'en souvient, L'vov croyait que les Russes étaient particulièrement capables de ce genre de démarche.

Il y avait pourtant, selon L'vov, deux domaines dans lesquels les Anglais et les Français auraient pu donner des leçons aux Russes.

«Si on voulait emprunter quelque chose dans l'art de la construction à telle ou telle nation, ce ne serait certainement pas les beautés, ni la proportion, ni le goût de l'Architecture grave (važnaja). Que les maçons anglais nous apprennent à construire de façon simple, propre et droite ; et que les architectes français nous apprennent à distribuer les intérieurs des maisons, car nous ne pouvons trouver chez les architectes anciens de ressemblance avec la vie moderne ( $p o$ obrazu nastojašč ǰžzizni). »49

On sait que, dans ses propres constructions, L'vov employait des maçons anglais, notamment ceux que Cameron avait fait venir en Russie. La première remarque pouvait donc refléter sa propre expérience pratique. Mais c'est surtout la seconde qui doit attirer notre attention. En effet, par ce commentaire, $L^{\prime}$ vov réduisait le goût palladien au domaine de l'architecture extérieure, c'est-à-dire aux façades, et en évacuait la distribution intérieure, la considérant comme du domaine français et par là banalisant toute sa reconstruction du «vrai goût palladien ». La propagande par les Modernes français (depuis Perrault jusqu'à Blondel) de la distribution «à la française », confortable et adaptée aux pays du Nord, a fait surgir au XVIII ${ }^{\mathrm{e}}$ siècle la célèbre formule : « les façades à l'italienne, les intérieurs à la française ». Les raisons de la réussite de cette stratégie moderne se trouvaient dans l'apparente évidence de ce constat : la distribution des pièces dans une maison devait servir les besoins du particulier et être donc adaptée autant que possible à sa façon de vivre, à ses mœurs et à ses usages. En conséquence, la distribution était considérée comme un domaine qui échappait à toute normalisation comme elle échappait également au souvenir de l'Antiquité. 
Par cette construction théorique, les Modernes français prétendaient contourner l'immense tentative des architectes de la Renaissance italienne, de Bramante à Pirro Ligorio, qui avaient visé à reconstruire la maison antique dans la structure du palazzo et de la villa, en se fondant sur les descriptions de Vitruve mais aussi sur les ruines de la Rome antique. Selon les humanistes italiens, la distribution des maisons ne devait pas suivre la façon de vivre et les caprices des particuliers en leur procurant le maximum de confort, mais au contraire, les formes architecturales intérieures empruntées aux Anciens devaient faire renaître la noblesse, la grandeur et la vertu héroïque des Romains ${ }^{50}$. Dans son traité d'architecture, Alberti évoquait déjà la Vertu comme, à la fois, la condition et le but suprême de toute œuvre architecturale. Pour Daniele Barbaro, auteur de l'édition de Vitruve à laquelle collabora Palladio, l'architecture était le modèle du perfectionnement de la nature humaine. C'est précisément dans son édition de Vitruve, « corrigée » par l'étude de 1'Antiquité, qu'apparaît pour la première fois la reconstruction palladienne de la maison privée des Anciens ${ }^{51}$.

Considérant que le temple antique avait hérité ses formes de la maison particulière, Palladio fonda sa reconstruction de la maison antique sur le modèle du temple, la dota d'un portique et la couronna d'une coupole. Dans ses distributions intérieures, la symétrie anthropomorphe, le système des proportions ainsi que la décoration étaient l'expression de sa foi en la nature divine de l'homme. Les ruines de la Rome antique étaient pour Palladio les témoignages des formes de vie nobles et vertueuses, supérieures à tout ce que l'on avait pu inventer depuis. L'imitation de la vertu antique était la tâche la plus profonde de son entreprise architecturale ${ }^{52}$. Ce n'est pas un hasard si le frontispice de ses Quattro libri comporte l'allégorie de la Regina Virtus, seule reine de la république vénitienne. Cette allégorie ne réapparaîtra dans aucune édition palladienne du XVIII ${ }^{\mathrm{e}}$ siècle. Comme nous l'avons vu, elle sera remplacée par une figure évoquant la Nation, ou encore par l'évocation iconographique de la Vérité.

50. Une littérature très importante est consacrée à cette question. Voir, par exemple : James S. Ackerman, « The Belvedere as a classical villa », Journal of the Warburg and Courtault Institutes, 14, 1951, p. 70-91 ; Vicenzo Fontana, «Raffaello e Vitruvio », in Vitruvio e Raffaello. II «De Architettura » di Vitruvio della traduzione inedita di Fabio Calvo Revennate, a cura di Vincenzo Fontana e Paolo Morachiello, Rome, Officina edizioni, 1975, p. 25-44 ; Christoph Luitpold Frommel, « Abitare all'antica : il Palazzo e la Villa da Brunelleschi a Bramante », in Rinascimento da Brunelleschi a Michelangelo. La rappresentazione dell'architetura, a cura di Henry Millon e Vittorio Magnago Lampugnani, Milan, Bompiani, 1994, p. 183-202 ; Maria Losito, Pirro Ligorio e il casino di Paolo IV in Vaticano, I' "essempio » delle « cose passate », Rome, Fratelli Palombi editori, 2000.

51. Vitruvio, I dieci libri dell'architettura tradotti e commentati da Daniele Barbaro (1567), con un saggio di Manfredo Tafuri (« La norma e il programma : il Vitruvio di Daniele Bararo ») e uno studio di Manuela Morresi, Milan, Edizioni il Polifilo, 1987.

52. Et pas seulement architecturale, d'ailleurs. Il s'agissait également pour Palladio de voir dans l'Antiquité le modèle de toute construction politique et civile : J. R. Hale, « Palladio, Polybius and Caesar », Journal of the Warburg and Courtauld Institutes, 40, 1977, p. 240255. 
Or le développement suivant, que L'vov consacre à la distribution intérieure chez Palladio, montre en fait toute la complexité de sa position face à ce problème fondamental :

«L'idée que les Anciens avaient de la beauté et de la majesté ne se conserve que dans les ruines des bâtiments de cette époque et ne s'accorde en rien à la nôtre. La magnificence ne résidait pas alors dans la qualité des choses, mais dans leur proportion et leur composition (obrazovanie), au lieu qu'à notre époque le luxe nous a appris à la trouver dans l'or, les riches tissus, les tapisseries, les décorations des maisons. Pompée, après avoir conquis l'Inde, employa tous ses trésors à construire un magnifique Amphithêâtre ; les Français vendraient certainement le matériau de ce dernier s'ils pouvaient aujourd'hui racheter les raretés indiennes pour décorer le boudoir ; mais dans ce boudoir, le Héros romain se serait senti à l'étroit et étouffé. Palladio, qui avait formé son goût sur les exemples de la magnificence ancienne, s'en tenait quelque peu, dans la distribution intérieure et dans les maisons particulières, à la symétrie sévère, à la gravité et à la simplicité, que l'Architecte de son siècle n'osait trahir sans opprobre ; c'est pourquoi aucun particulier des temps modernes ne trouvera confortable (pokojnyj) la distribution intérieure de ses maisons. Palladio, qui avait en son temps une parfaite idée de la maison avantageuse et confortable, n'avait pu prévoir par une vision prophétique les besoins et les caprices des hommes nés 200 ans après lui. Des garde-robes (ubornyja), des cabinets pour le repos, des divans [?] et autres petits compartiments ne comptaient pas parmi les pièces (pokol) ${ }^{53}$; les plans des maisons qui, dans leur simplicité, avaient quelque chose de majestueux sous le rapport de l'art, offraient un logement beau et confortable aux hommes de mœurs simples. Les grands seigneurs étaient contents quand on leur construisait des salles pour les exercices de la guerre ${ }^{54}$ et pour les portraits de leurs ancêtres, une bibliothèque, une galerie, une grande antichambre (prihožaja), un magnifique vestibule (seni). Il faut avouer que dans la distribution des pièces selon le goût de son époque, Palladio était décidément supérieur à tous les autres Architectes de son temps; il s'était d'autant plus distingué parmi eux qu'il était seul à connaître le mystère grâce auquel on communique aux habitations des hommes particuliers la magnificence et la gravité (važnost) des bâtiments publics romains. Cette rare qualité distingue ses constructions de tous les autres bâtisseurs de son siècle. Tel, bien sûr, doit être l'Architecte qui fonde sa doctrine sur les règles de Vitruve et sur les exemples de l'Architecture ancienne. $^{55}$

D'une part, donc, L'vov reproduit ici la conviction des Modernes français selon laquelle la distribution intérieure doit suivre les pratiques des différents pays et des différentes époques, comme une sorte de costume qui obéit à la mode. Ainsi l'expérience palladienne perd sa valeur idéale et intemporelle : Palladio se transforme en un architecte de son époque, encore que le meilleur, qui a adopté dans ses distributions intérieures les goûts de son temps. D'autre part, ces goûts de l'époque de Palladio,

53. Note de L'vov: «Cet arrangement bisarre auroit eu l'air d'un labyrinthe et un bâtiment construit selon cette méthode eut été regardé plutot comme une ruche d'abeilles que comme une habitation des hommes. Bâtimens et dessins d'André Pallađio par Scamozzi » (en français dans le texte).

54. Note de L'vov : « Même auteur, et sur la même page » (en français dans le texte).

55. « Ot izdatelja ruskago Palladija », art. cit. 
simples et nobles, sont décrits avec la sympathie la plus vive et la plus profonde. Plus encore, L'vov est parfaitement conscient que ces goûts mêmes ont été formés sur un grand modèle, celui de l'Antiquité romaine, qu'ils n'appartiennent donc pas complètement à son époque, mais qu'ils ont une valeur humaine universelle. Le fait que les habitations des hommes particuliers recevaient chez Palladio la magnificence et la gravité des bâtiments publics romains est signifié dans le texte de L'vov par le mot «mystère » (tainstvo), très fort par ses connotations religieuses.

Cette ambiguïté de L'vov dans la question de la distribution provient sans doute de sa source d'inspiration qui, paradoxalement, n'est pas française, mais italienne, à savoir la théorie architecturale vénitienne du XVIII ${ }^{\text {e }}$ siècle, celle de Tommaso Temanza ${ }^{56}$, dont il reprend dans sa préface la biographie de Palladio ${ }^{57}$, ou encore de Bertotti Scamozzi ${ }^{58}$, dont il cite la célèbre édition des bâtiments de Palladio. Malgré la complexité de la pensée de Temanza qu'il nous est impossible de développer ici, et malgré la profonde différence qui existe entre lui et Scamozzi ${ }^{59}$, nous pouvons relever chez ces deux palladianistes un point commun. En réponse à la critique française, de plus en plus persistante, des distributions italiennes en général et palladiennes en particulier, les deux architectes soulignent que les maisons palladiennes étaient commodes pour les Vénitiens de son époque. Il font done de Palladio l'architecte de son siècle, qui adopta ses bâtiments aux usages des commanditaires.

Pourtant, la pensée de L'vov ne se réduit pas à la reproduction des maximes de la théorie architecturale vénitienne de XVIII ${ }^{e}$ siècle. Pour mieux comprendre son originalité, il faut bien savoir qu'on pouvait tirer de Palladio aussi bien une théorie puriste, en partie celle de Temanza, mais surtout celle de Francesco Miliza ${ }^{60}$, que le sentiment de la liberté qui s'acquiert grâce à l'intime connaissance de l'Antiquité. Dans les commentaires de L'vov, nous voyons apparaître de temps en temps les

56. Voir par exemple l'une des dernières publication sur Temanza : Patrizia Valle, Tommaso Temanza e l'architettura civile. Venezia e il settecento: diffusione e funzionalizzazione dell' architettura, Rome, Officina Edizioni, 1989.

57. Vita di Andrea Palladio vicentino egregio architetto scritta di Tommazo Temanza architetto, ed ingegnere della serenissima repubblica di Venezia, Accademico olimpico di Vicenza, et ricovrato di Padova aggiuntevi in fine due scritture dello stesso Palladio finora inedite, Venise, presso Giambatista Pasquali, 1762 (rééd. dans: Vite dei più celebri architetti e scultori vanaziani...,Venise, 1778, p. 284-408). Cette biographie de Palladio est la source principale de L'vov. Mais il cite également la première biographie de Palladio par Paulo Gualdo : in Giovanni Montenari, Del Teatro olimpico di Andrea Palladio in Vicenza..., Padoue, G. Conzatti, 1733 (rééd. par G. G. Zorzi, dans Saggi e memorie di Storia dell'arte, II, 1959, p. 93-104.)

58. J. Quentin Hughes, Introduction, in Le fabbriche e i disegni di Andrea Palladio, raccolti ed illustrati da Ottavio Bertotti Scamozzi, Vicence, 1796. Rééd. Londres, Alec Tiranti, 1968. De ce même milieu, L'vov mentionne dans sa préface le comte Alessandro Pompei, auteur du livre Li cinque ordini dell'architettura civile di Michele Sanmicheli, Vérone, 1735. Sur Pompei voir : Camillo Semenzato, «Un architetto illuminista : Alessandro Pompei », Arte veneta, 15 , 1961, p. 192-200.

59. Voir notamment: L. Olivato, Ottavio Bertotti Scamozzi studioso di Andrea Palladio, Vicence, 1976 ; Id., « Une relazione difficile. Lettere inedite di Tommazo Temanza a O. B. Scamozzi », Arte veneta, 33, 1979, p. 169-173.

60. Giulio Carlo Argan, «Andrea Palladio et la critica neo-classica », Arte, juillet 1930, p. $327-$ 346. 
éléments de la critique puriste et rigide dont L'vov pouvait voir l'exemple chez Temanza. Ainsi s'acharne-t-il contre les «abus » de Palladio ${ }^{61}$. En évoquant l'entrecolonnement central que Palladio a fait plus large que les autres, $L$ 'vov écrit :

« Dans ce cas Palladio ne respectait pas entièrement la règle des architectes grecs qui, dans leurs constructions magnifiques, nous ont laissé peu d'exemples d'entrecolonnement central plus large que les autres. Le temple de Thésée d'Athènes, les temples de Paestum, le Parthénon, et d'autres célèbres vestiges des bâtiments d'Athènes témoignent de cette vérité. Dans ce cas, Palladio incite à sacrifier la beauté de la composition générale, si agréable à la vue, aux besoins de mieux voir l'entrée et ses décorations. Mais pour cela il ne me semble pas nécessaire de réduire l'entrée ou d'écarter les colonnes, car le champ de vue s'élargit quand nous nous approchons de l'objet, et celui qui entre dans le temple verra toujours de loin les deux parties de la porte, même si elle sont fermées des deux côtés par un quart de colonne. Il ne faudrait faire l'entrecolonnement central plus large que les autres que si les colonnes sont placées contre les murs, ou encore, que Dieu nous en préserve, si elles sont posées dans le mur. »62

L'vov « corrige » donc Palladio par l'architecture grecque et redoute par ailleurs le procédé typiquement baroque - les colonnes engagées dans les murs.

Pourtant, tout en mettant en garde contre les « abus » de Palladio, L'vov revendique une totale liberté pour l'architecte qui se nourrit de l'Antiquité. Ainsi il commente la réflexion palladienne sur les proportions de l'ordre dorique :

«En prescrivant des règles rigoureuses et des mesures communes à chaque ordre, l'art a indubitablement aidé les élèves et les artistes médiocres, mais en revanche, il a posé des bornes aux talents supérieurs et au goût. Buonarotti aimait à dire que le véritable compas d'un bon architecte était son oil. Les ruines des bâtiments antiques, seuls flambeaux justes menant l'artiste à la vraie magnificence et au bon goût, confirment la vérité du mot de ce grand homme. Parmi les temples antiques d'un même ordre, on n'en trouvera presque jamais deux qui aient les mêmes proportions ; l'emplacement des bâtiments n'était pas seul à déterminer ces différences de proportions, leur usage même en était la raison. Un temple dorique d'Hercule avait sept modules et demi ; les portes d'une ville du même ordre en avaient six et demi. L'un représentait la virilité et la beauté, tandis que les autres ne devaient représenter que la virilité et la solidité. Ce commentaire ne sera peut-être pas inutile à l'artiste qui s'écartera des sentiers battus quand il voudra éprouver la théorie sur des exemples tirés des ruines de l'architecture antique. » ${ }^{63}$

Cette façon de penser, qui rappelle les commentaires de Daniele Barbaro sur le texte vitruvien ou encore la façon de voir et de faire de Bernini, est très proche de l'esthétique de Piranesi ${ }^{64}$, de Clérisseau, de Peyre, des frères Adams et de

61. Čtyre knigi palladievoj arhitektury, op. cit., note 19 , p. 32.

62. Ibid.

63. Ibid., note 21 , p. 37-38.

64. Voir : Rudolf Wuttkower, « Piranesi's "Parere su l'architettura" », Journal of the Warburg and Courtauld Institutes, 2, 1938-1939, p. 147-173. 
Quarenghi qui étaient tous très liés à Rome dans les années 1750-1760. Dans les cuvres architecturales ainsi que dans les écrits de ce dernier nous trouvons des idées très proches du passage de L'vov que l'on vient de citer.

Il nous semble de façon générale que la lecture lvovienne de Palladio doit être mise en rapport avec la présence en Russie de Giacomo Quarenghi65. On sait que les deux hommes furent unis par un travail commun sur l'édition de l'album pour le couronnement de Paul Ier66, ainsi que par le projet de la maison et du parc de Bezborodko à Moscou. Il est intéressant de remarquer que dans ses commentaires, lorsqu'il veut donner des exemples de la magnificence romaine, L'vov mentionne le même monument - le théâtre de Pompée - que donne en même temps Quarenghi dans la description de son théâtre de l'Ermitage.

Cette même attitude non dogmatique et non puriste, libre et artistique, pénètre le style des gravures de l'édition de L'vov que l'on doit également, nous semble-t-il, attribuer à l'influence de Quarenghi, lui-même brillant dessinateur. Les gravures de $\mathrm{L}^{\prime} \mathrm{vov}^{67}$ [ill.9 et 11] qui agrandissent les gravures originales de Palladio [ill. 8 et 10] sont exécutées dans la technique du mezzo-tinto, qui reproduit les effets du clairobscur dans un dessin libre lavé à l'encre ou à l'aquarelle jaunâtre. Pour comprendre cette façon de reproduire les gravures de Palladio, il nous faut revenir à la biographie de Palladio par Temanza, collectionneur des dessins de Palladio et ami proche de Quarenghi : «Palladio fut un excellent dessinateur de toutes sortes de choses. Dans ses aquarelles il utilisait une certaine teinte jaune. ${ }^{68}$ Dans son édition, L'vov semble donc vouloir imiter plutôt les dessins de Palladio que les

65. Sur Quarenghi voir par exemple : Gracomo Quarenghi. Architetto a Pietroburgo. Lettere e altri scritti, A cura di Vanni Zanella, Venise, Albrizzi editore, 1988 ; Monumenta bergomensia XXI. I cinque album di Giacomo Quarenghi nella Civica Bibilioteca di Bergamo, a cura di Sandro Angelini, Bergame, 1967 ; Giacomo Quarenghi, a cura di Sandro Angelini, testo di Vladimir Pilavskij, catalogo di Vanni Zanella, Bergame, Edizione dal Credito Bergamasco, 1984 ; Giacomo Quarenghi. Architetture e vedute, Milan, Electa, 1994 ; Piervaleriano Agelini, Miliza Korzunova, Giovanna Nepi Scirè, Disegni di Giacomo Quarenghi. Vedute e capricci, Galerie dell'Accademia di Venezia, Milan, Electa, 1996 ; I disegni di Giacomo Quarenghi al Castello Sfonzesco, Venise, Marsilio Editori, 1998 ; Voir également : Giacomo Quarenghi e il suo tempo, atti del convegno, a cura di Silvia Burini, Bergame, Moretti \& Vitali editori, 1995. En langue russe voir par exemple : Milica Koršunova, Džakomo Kvarengi, Leningrad, 1977 ; Viktor Graščenkov, « Džakomo Kvarengi i venecianskij neoklassicizm » (Giacomo Quarenghi et le néo-classicisme vénitien), Sovetskoe iskusstvoznanie, 20, 1986, p. 301-366; Id. «Džakomo Kvarengi i arhitektura evropejskogo neoklassicizma » (Giacomo Quarenghi et l'architecture du néo-classicisme européen), Rossija i Italija. Vstreča dvuh kul 'tur, (Moscou), 4, 2000, p. 69-84 ; A. Pavelkina, Giacomo Quarenghi, catalogue de l'exposition, Musée de l'Histoire de Saint-Pétersbourg, 1998, Saint-Pétersbourg, 1998 ; Džakomo Kvarengi. Arhitekturnaja grafika, catalogue de l'exposition, Ermitage, Saint-Pétersburg, 1999.

66. Milica Koršunova, « Dž, Kvarengi i N. A. L’vov. Sovmestnaja rabota v svjazi s koronaciej Pavla I » (G. Quarenghi et N. A. L'vov. Leur travail commun à l'occasion du couronnement de Paul I ${ }^{\mathrm{er}}$ ), in Zarubežnye hudožniki i Rossija (Les artistes étrangers et la Russie), Saint-Pétersbourg, 1991, p. 56-61.

67. On lit sur certaines gravures : «Sculpt. N. Lwoff, direx. N. Lwoff. » D'autres ont été gravées par J. C. Nabholz.

68. Tommazo Temanza, «Vita di Andrea Palladio », in : Vite dei più celebri architetti e scultori vanaziani..., Venise, 1778 , p. 391. 
gravures linéaires et assez sèches de son édition, voire même les rendre encore plus « vivants ${ }^{69}$.

Il est vrai que, dans ses commentaires, L'vov souligne à plusieurs reprises que les distributions palladiennes ne conviennent pas aux conditions climatiques de la Russie :

« Palladio lui-même ne suivait pas toujours cette règle, fondée sur la symétrie des corps, qu'il adaptait parfois pour la commodité de l'existence ; sans cela, de nombreux propriétaires auraient considéré leurs maisons comme une hydre éternelle qui les torturait sans cesse et qui ne vieillissait pas, ils auraient attendu avec impatience une fissure quelconque qui aurait annoncé la nécessité de les reconstruire. En Italie, on peut certainement construire des maisons en suivant le plan d'un échiquier. Là-bas, le maître occupe souvent un petit coin de sa maison, tandis que dans les autres pièces, les tableaux et les marbres ne craignent pas le froid. Les Italiens ignorent les courants d'air, en hiver la température de leurs appartements est la même qu'au dehors; en été, au dehors comme dans les appartements, les fenêtres et les portes sont ouvertes comme des grilles; et cependant, nous allons chez eux pour nous réchauffer. Ainsi, la règle de l'équilibre nous convient-elle? Et quel équilibre ne serait corrigé par un froid de moins 28 degrés ? 70

Il est vrai aussi que dans ses textes il exalte à plusieurs reprises les traits spécifiques que l'architecture en général et l'art des jardins en particulier devraient adopter selon lui sous l'influence du climat russe. Ce type de réflexions correspond exactement à la critique française ${ }^{71}$. Pourtant L'vov construit lui-même plusieurs maisons dans les propriétés des nobles, dont la sienne, selon la distribution palladienne. De façon générale, le type de «villa » qu'il pratique dans les années 1780-1790 est parfaitement palladien : bloc cubique, salle centrale recouverte d'une coupole, portique ou loggia. Une étroite relation entre l'architecture palladienne et les formes de vie dans une « villa » surgit dans l'inscription sur la gravure qui représente la maison de L'vov ${ }^{72}$ : «Maison dans le village de Čerenčicy, à 15 verstes de Toržok. Projetée, dessinée, coloriée, construite, gravée et habitée par Nikolaj L'vov $\gg^{73}$. Par ailleurs la correspondance de L'vov avec Deržavin à propos de la vie aux champs est remplie de topoi antiques. La profonde compréhension par Deržavin de l'esprit palladien apparaît bien dans la formule poétique qu'il donne de sa maison construite par L'vov : «moj hramovidnyj dom » (ma maison semblable au temple).

69. Voir Giangiorgio Zorzi, I disegni delle antichita di Andrea Palladio, Venise, Neri Pozza editore, 1959.

70. Četyre knigi Palladievoj arhitektury, op. cit., note 32, p. 58.

71. Jean-Marie Pérouse de Montclos, «Palladio et la théorie classique dans l'architecture française du XVII siècle », Bollettino CISA, XII, 1970, p. 97-105.

72. Musée du palais Voroncov à Alupka.

73. Cité par M. V Budylina et al., op. cit.,p. 14. 
Enfin dans son projet du parc de Bezborodko qui est chronologiquement très proche de l'édition de Palladio (1797-1798) ${ }^{74}$ et qu'il réalise en étroite collaboration avec Quarenghi, L'vov suit le modèle de la Renaissance italienne. Quarenghi projette pour Bezborodko une maison presque entièrement occupée par le théâtre et les galeries de peintures, c'est-à-dire une maison proche de l'idéal palladien selon la description lvovienne. De son côté, $L^{\prime}$ vov prétend dans son jardin « faire renaître la magnificence des antiques jeux de gymnase». En s'inspirant de la villa d'Hadrien, il place dans son jardin à l'antique un amphithéâtre, une naumachie, un lycée, un hippodrome. Ces formes ne doivent surtout pas rester stériles ni se réduire à la décoration. Il faut au contraire que les jeux soient réellement pratiqués dans ces lieux, sinon ils seront morts et inutiles. Ainsi L'vov partage la conviction des architectes de la Renaissance italienne, selon laquelle l'architecture peut être un moyen de faire renaitre les pratiques antiques. Cette conviction, Quarenghi et L'vov la transmettent au commanditaire qui écrit en novembre 1797 à Semen Voroncov: «J'ai décidé d'entreprendre un nouveau bâtiment qui, du moins, montrera aux futures générations qu'à notre siècle et dans notre pays on savait ce que c'est que le goût (znali vkus). »75

Le « vrai goût » palladien que L'vov voudrait atteindre est en réalité une idée, un rêve de l'architecture qui divinise l'homme, qui, aussi bien extérieurement qu'intérieurement, le fait remonter vers la suprême noblesse et la vertu. À travers ce rêve transparaît l'idéal cicéronien, qui réapparaît à l'époque de L'vov, notamment dans les écrits sur l'architecture d'Algarotti qui compare l'architecture à la métaphysique ${ }^{76}$. Ainsi L'vov écrit dans un de ses commentaires :

«Quoique les frontons décoratifs ne protègent pas les habitants des intempéries, ils n'en protègent pas moins la partie à laquelle ils servent de couverture; et même s'il n'en est pas besoin, ils doivent au moins en donner l'image. Seul le décor qui inspire l'idée du nécessaire est à sa place ; les petits motifs décoratifs (kružki, krjučki $i$ vpadinki) figurant sur un bâtiment bien proportionné ne le décorent pas plus que des pièces de brocart ne décorent un cafetan bien coupé et simple. $\gg^{77}$

Il nous est facile de reconnaître la voix de Cicéron derrière celle de L'vov :

«Ce fronton du Capitole et ceux des autres temples sont le produit non de la beauté mais de l'inéluctable nécessité ; car ce fut en calculant comment évacuer

74. G. G. Grimm, « Proekt sada Bezborodko v Moskve... », art. cit. L’album intitulé « Sad knazja Bezborodko pri moskovskom dome » se trouve au Musée de l'Académie des beaux-arts à Saint-Pétersbourg (NIMAH), n ${ }^{\circ} \mathrm{KP}$ 100/3. Il contient les 11 dessins et une explication en russe et en français : Kakim obrazom dolžno by bylo raspoložit' sad knjazja Bezborodki $v$ Moskve. De quelle façon il conviendrait de disposer le jardin du prince Bezborodko à Moscou.

75. Ibid., p. 128.

76. Francesco Algarotti, Saggio sopra I'architettura (1762) éd. par Giovanni da Pozzo, Bari, Latezza, 1963.

77. Čtyre knigi Palladievoj arhitektury, op. cit., note 29 , p. 55-56. 
les eaux pluviales des deux côtés du toit qu'on obtint ce noble dessin des pignons comme résultante des nécessités de la structure - à tel point même que, si l'on édifiait une citadelle dans les cieux, où nulle pluie ne pourrait tomber, on jugerait infailliblement qu'elle manque tout à fait de noblesse sans fronton. ${ }^{78}$

Ainsi L'vov suit ici l'idéal de l'architecture «à l'antique » qui ne dépend ni de l'époque, ni du climat. Il est en revanche capable de résister aux petits « goûts » provinciaux du temps et de la nation. Or le conflit entre ces deux visions de l'architecture est presque irréductible. L'architecture est un art bien particulier : par sa finalité pratique, elle court constamment le risque de perdre son statut d'art libéral pour lequel les humanistes italiens avaient tant combattu. Comme nous venons de le voir, L'vov était conscient de cette contradiction. Malgré ses ambiguïtés - ou peut-être grâce à elles - qui reflètent ces tensions dramatiques, il réussit à créer un livre dans lequel on entend parfaitement l'écho du « vrai goût palladien ».

Centre d'études du monde russe, soviétique et post-soviétique

54 , boulevard Raspail

75006 Paris

o.medvedkova@libertysurf.fr

78. Cicéron, De Oratore, III, 180. 